Journal of Theoretical and Applied Mechanics, Sofia, Vol. 47 No. 4 (2017) pp. 48-74

\title{
TORSIONAL WAVE PROPAGATION IN A PRE-STRESSED STRUCTURE WITH CORRUGATED AND LOOSELY BONDED SURFACES
}

\author{
Manoj K. Singh*, SAnjeev A. SAhU \\ Indian Institute of Technology (Indian School of Mines), \\ Dhanbad-826004, Jharkhand, India
}

[Received 19 April 2017. Accepted 4 December 2017]

\begin{abstract}
An analytical model is presented to study the behaviour of propagation of torsional surface waves in initially stressed porous layer, sandwiched between an orthotropic half-space with initial stress and pre-stressed inhomogeneous anisotropic half-space. The boundary surfaces of the layer and halfspaces are taken as corrugated, as well as loosely bonded. The heterogeneity of the lower half-space is due to trigonometric variation in elastic parameters of the pre-stressed inhomogeneous anisotropic medium. Expression for dispersion relation has been obtained in closed form for the present analytical model to observe the effect of undulation parameter, flatness parameter and porosity on the propagation of torsional surface waves. The obtained dispersion relation is found to be in well agreement with classical Love wave equation for a particular case. The cases of ideally smooth interface and welded interface have also been analysed. Numerical example and graphical illustrations are made to demonstrate notable effect of initial stress, wave number, heterogeneity parameter and initial stress on the phase velocity of torsional surface waves.
\end{abstract}

KEY WORDS: Corrugation, loose bonding, dispersion, orthotropic, heterogeneity

\section{LIST OF SYMBOLS}

$K$ is the effective bonding parameter;

$k$ is the wave number;

$\varsigma$ is the pure real number;

$g_{1}(r)$ is the corrugated boundary surfaces of the porous layer;

$g_{2}(r)$ is the corrugated boundary surfaces of lower the half-space;

$H$ is the thickness of the layer;

$2 \pi / b$ is the wavelength of corrugation;

$\kappa$ is the amplitude of corrugation;

${ }^{*}$ Corresponding author e-mail: manojmathism@gmail.com 
$\chi_{i j}$ are the stress components of upper half-space;

$A_{i j}$ are the elastic constants for upper half-space;

$\nu_{i j}$ are the strain components for the upper half-space;

$w_{r}^{(1)}, w_{\theta}^{(1)}, w_{z}^{(1)}$ are the components of displacement for the upper half-space;

$D, F$ are the rigidity of upper half-space along radial and axial direction;

$P$ is the initial stress for the upper half-space;

$\beta_{1}$ is the shear wave velocity for the upper half-space;

$J_{1}(k r)$ is the Bessel's function of first order;

$\varpi_{i j}$ are the components of stress for the porous layer;

$w_{r}^{(2)}, w_{\theta}^{(2)}, w_{z}^{(2)}$ are the components of solid phase displacements for the porous layer;

$W_{r}, W_{\theta}, W_{z}$ are the components of fluid phase displacements for the porous layer;

$P_{1}$ is the initial stress for the porous layer;

$u_{r}, u_{\theta}, u_{z}$ are the rotational components for the porous layer;

$\alpha_{i j}$ are the strain components for the porous layer;

$p_{f}$ is the pressure in the fluid of porous layer;

$\pi$ is the porosity of the poroelastic layer;

$\rho_{r r}, \rho_{r \theta}, \rho_{\theta \theta}$ are the mass coefficients;

$\rho^{(2)}$ is the density the solid-liquid for the porous layer;

$p_{s}, p_{f}$ are the mass densities of the solid and liquid for the porous layer;

$N^{\prime}, L$ are the rigidities of the porous layer, along radial and axial direction;

$Q$ is the porosity parameter for the porous layer;

$\beta_{2}$ is the shear wave velocity of porous layer;

$\tau_{i j}$ are stress components for the lower inhomogeneous anisotropic half-space;

$\alpha$ is the heterogeneity parameter for the lower half-space;

$P^{\prime \prime}$ is the initial stress for the lower half-space;

$\rho_{3}$ is the density of the lower medium;

$\beta_{3}$ is the shear wave velocity for the lower half-space;

$L^{\prime}, A$ are the rigidity of the lower half-space along radial and axial direction;

$\Omega_{1}$ is bonding parameter of common interface of upper half-space and intermediate layer;

$\Omega_{2}$ is the bonding parameter of common interface intermediate layer and lower half-space;

$\kappa_{1}$ is the flatness parameter for the intermediate layer;

$\kappa_{2}$ is the flatness parameter for the upper half-space;

$g_{1}(r)=0$ represents plane boundary surface of the upper half-space;

$g_{2}(r)=0$ represents plane boundary surface of the lower half-space. 


\section{INTRODUCTION}

It is well known fact, that the boundary surface of the Earth is not regular. There are different types of irregularity in shapes and sizes are found inside the Earth. Many authors have studied the boundary surface of the Earth by taking parabolic, hyperbolic and corrugated boundary surface and also some complicated structure in their research works. The propagation of different type's wave inside the Earth is very much affected by these types of irregularity. Selim [1] discussed the static deformation of an irregular initially stressed medium. Seismic waves through a layered half-space with corrugated boundaries were investigated by Zhang and Shinozuka [2]. Chen [3] visualized the reflection/transmission matrices method for solving the generation and propagation of a seismic SH wave in multilayered media with irregular interfaces. Singh and Tomar [4] studied the qP-wave at a corrugated interface, between two dissimilar pre-stressed elastic half-space. Love wave behaviour at a layer medium, bounded by irregular boundary surfaces, is studied by Singh [5]. Apart from these, some problem on the reflection and refraction at corrugated surface is explained by Rayleigh [6] and Asano [7]. The propagation of torsional waves in elastic layered media is highly interested to seismologist and earth science, due to its property of twisting the particle in direction of wave motion. Torsional surface waves in inhomogeneous elastic media was discussed by Vardoulakis [8]. Later on Georgiadis [9] et al. have explained propagation of torsional surface waves in gradient elastic half-space. Vishwakarma et al. [10] have presented the torsional surface wave in a homogeneous crustal layer over a viscoelastic mantle. Torsional surface waves in inhomogeneous elastic media was discussed by Vardoulakis [11]. The propagation of waves in elastic media, related to initial stress, has become interesting in various fields such as construction, retaining structures and embankments. The initial stress is termed as stresses, which exist in an elastic body, without action of some external forces. The motive for considering initial stress in medium is due to many reasons, for example the resulting effect of atmospheric pressure, temperature difference etc. Therefore, it is much delightful to highlight the influence of these initial stress on the propagation of waves. Effect of initial stress on the propagation behaviour of Love waves in a layered piezoelectric structure was presented by Liu [12] et al. Qian et al [13] have discussed propagation of Love waves in a piezoelectric layered structure with initial stresses. Biot [14] has delineated the influence of initial stress on elastic waves. Influence of gravity and initial stress on the Love waves in a transversely isotropic medium was studied by Dey and Chakraborty [15].

There are some materials, in which mechanical and thermal property are unique, called orthotropic materiels, as wood coled-roll. Orthotropic material exhibits different material properties along three perpendicular axes. Akbarov and Ilhan [16] 
investigated the dynamics of a system, comprising a pre-stressed orthotropic layer and pre-stressed orthotropic half-plane, under the action of a moving load. The earth model is more likely to be porous and hence, the loose boundaries cannot be ruled out. In fact, this may be a more realistic case. The concept of poroelastic material has been used in various fields of applied science and engineering, such as soil and rock mechanics, hydrogeology, acoustics, filtration, construction engineering, biophysics and material science. In recent past significant works have been carried out in the direction to analyses the propagation of surface waves in anisotropic composites, involving porous medium, few of them are Dai et al. [17, 18], Ke et al. [19], Son, Kang [20]. Contributing a bit advance to the state of art of surface wave analysis, Singh and Lakshman [21] have recently pointed out the effect of loosely bonded undulated boundary surfaces of doubly layered half-space on the propagation of torsional type of surface wave. They considered the model comprise of fiber-reinforce, porous and viscoelastic media, such that the porous layer is sandwiched between the rest two. Following the similar methodology, we have extended our effort to highlight the wave behaviour in a more complex mechanical structure, where the porous layer is bonded between orthotropic and heterogeneous isotropic semi-infinite media. Stress, already present in all three media (termed as initial stress), has been considered as the integral part of the structure. A valuable information may be encountered by comparing the result(s) of present paper with that of the article by Singh and Laxman [21], that the increment in initial stress parameter acting in porous layer decreases the phase velocity of torsional wave in their structure whereas the same parameter, acting in porous layer is found responsible to increase the phase velocity of torsional wave in present paper.

The motive of the present paper is to investigate the behaviour of torsional wave in a corrugated, as well as, loosely bonded poroelastic layer, sandwiched between an orthotropic half-space, under initial stress and pre-stressed inhomogeneous anisotropic half-space. Using the mathematical methods, dispersion relation has been obtained in closed form which specify the dependence of phase velocity over the wave number. Dispersion curves are plotted to highlight the effect of corrugated boundary surfaces, porosity, initial stress, and heterogeneity on the propagation of torsional wave. Notable effect of wave number, undulation parameter, flatness parameter and bonding parameter on the propagation of torsional wave, have been described. Obtained result has been matched with classical Love wave equation, as a particular case of the considered problem.

\section{BASIC HYPOTHESIS}

Murty $[22,23]$ defined a single real bonding parameter to which numerical values can be assigned, corresponding to a given degree of bonding between two half-spaces 
and particularly, he showed that the acoustic behaviour of ideally smooth and fullybonded interfaces to correspond to values 0 and $\infty$ of the bonding parameter, respectively. Murty carried his study by considering two fundamental assumptions. The first assumption demonstrate, that loosely bonded surface between two elastic halfspace assures the continuity across the interface. The second assumption is that slip at interface is proportional to the local shearing stress, i.e.:

$$
\text { Shearing stress }=K \times \text { Slip. }
$$

Therefore, vanishing of $K$ (the proportionality constant) corresponds to an ideally smooth interface and an infinitely large value of $K$ corresponds to a welded interface. The intermediate values of $K$ represent a loosely bonded interface. Moreover, the effective bonding parameter $K$ can be redefined in a more appropriate form (Singh et al. [24]):

$$
\text { Shearing stress }=i \varsigma K \mu\left(v-v^{\prime}\right) \text {, }
$$

where $\varsigma$ is the pure real number, $\mu$ is the rigidity of the lower layer and $v, v^{\prime}$ are displacement components for upper and lower layer. Further, the dimensions on the two sides of Eq. (2) demand that as $K$ occurs there, it cannot be a pure real number. Now we introduced a new real variable $\Omega$, such that:

$$
\varsigma=\frac{c_{0}}{\beta_{0}} \frac{\Omega}{1-\Omega},
$$

where $c_{0}$ is the phase velocity of wave and $\beta_{0}$ is the shear wave velocity of the layer. Hence:

$$
\text { Shearing stress }=i \frac{c_{0}}{\beta_{0}} \frac{\Omega}{1-\Omega} K \mu\left(v-v^{\prime}\right) \text {, }
$$

Therefore, the value of $\Omega=0$ corresponds to an ideally smooth interface and when $\Omega=1$, then shearing stress is infinitely large, which represents the interface of medium to be welded.

\section{FORMULATION AND GEOMETRY OF THE PROBLEM}

To analyse the present problem geometrically, we have taken cylindrical coordinate system in such a way that origin, $o$ is at the common interface of the layer and the lower half-space, $r$-axis is along the direction of propagation of torsional wave and $z$-axis is pointing vertically downward. $g_{1}(r)$ and $g_{2}(r)$ are continuous functions of 
Torsional Wave Propagation in a Pre-Stressed Structure with Corrugated and ... 53

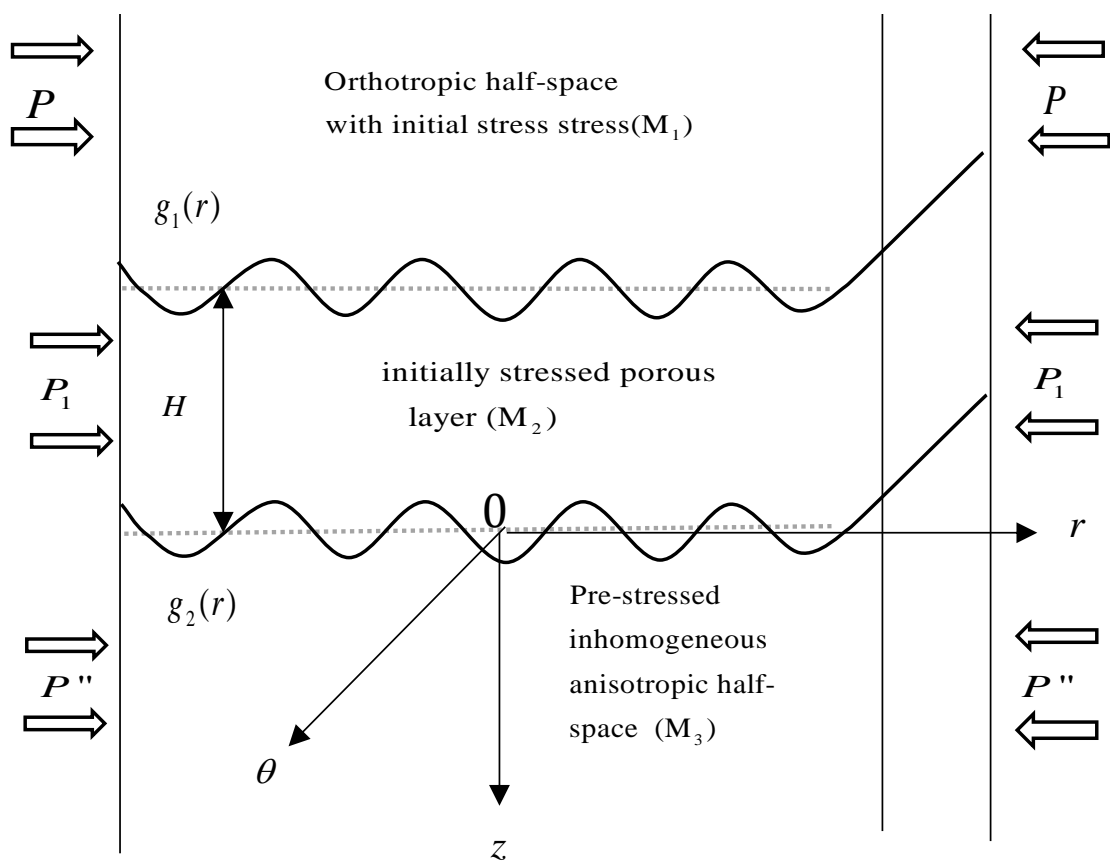

Fig. 1. Geometry of the problem.

independent of $\theta$ representing corrugated boundary surfaces of the layer and halfspace, as shown in Fig. 1, $H$ is the thickness of the layer. Nature of these functions is periodic and can be represented in Fourier series expansion, as follows (Asano [7]):

$$
g(r)=\sum_{n=1}^{\infty}\left(g_{n} \mathrm{e}^{i n b r}+g_{-n} \mathrm{e}^{-i n b r}\right),
$$

where $g_{n}$ and $g_{-n}$ are Fourier series expansion coefficients and $b$ is the wave number of corrugated surface, so that wavelength of corrugation is $2 \pi / b, n$ is the series expansion order. Introducing the constants $\kappa, p_{n}$ and $q_{n}$, as follows:

$$
g_{1}=g_{-1}=\frac{\kappa}{2}, \quad g_{ \pm n}=\frac{p_{n} \mp i q_{n}}{2}, \quad n=2,3,4 \ldots
$$

In view of eq. (5), eq. (1) results in:

$$
\begin{aligned}
g=\kappa \cos b r & +p_{2} \cos 2 b r+q_{2} \sin 2 b r \\
& +p_{3} \cos 3 b r+q_{3} \sin 3 b r+\cdots p_{n} \cos n b r+q_{n} \sin n b r+\cdots
\end{aligned}
$$


Now, if we consider the above expansion up to first order, then the interface shape can be expressed by only one cosine term, i.e., $g=\kappa \cos b r$, where $2 \pi / b$ is the wavelength of corrugation and $\kappa$ is the amplitude of corrugation (known as flatness parameter).

\section{GOVERNING EQUATIONS AND SOLUTION FOR THE UPPER HALF-SPACE}

The upper semi-infinite medium is considered as orthotropic medium under initial stress along $r$-axis. Then the equation of motion under initial stress in the absence of body forced is given by:

$$
\frac{\partial \chi_{r \theta}}{\partial r}+\frac{\partial \chi_{\theta z}}{\partial z}+\frac{2}{r} \chi_{r \theta}-\frac{\partial}{\partial z}\left(\frac{P}{2} \frac{\partial \chi_{\theta}^{(1)}}{\partial z}\right)=\rho_{1} \frac{\partial^{2} \chi_{\theta}^{(1)}}{\partial t^{2}}
$$

where $P$ and $\rho_{1}$ is the initial stress and density of the upper orthotropic half-space. The stress-strain relation for the upper orthotropic half-space is:

$$
\left[\begin{array}{c}
\chi_{r r} \\
\chi_{\theta \theta} \\
\chi_{z z} \\
\chi_{\theta z} \\
\chi_{r z} \\
\chi_{r \theta}
\end{array}\right]=\left[\begin{array}{cccccc}
A_{11} & A_{12} & A_{13} & 0 & 0 & 0 \\
A_{12} & A_{22} & A_{23} & 0 & 0 & 0 \\
A_{13} & A_{23} & a_{33} & 0 & 0 & 0 \\
0 & 0 & 0 & 2 D & 0 & 0 \\
0 & 0 & 0 & 0 & 2 E & 0 \\
0 & 0 & 0 & 0 & 0 & 2 F
\end{array}\right]\left[\begin{array}{c}
\nu_{r r} \\
\nu_{\theta \theta} \\
\nu_{z z} \\
\nu_{\theta z} \\
\nu_{r z} \\
\nu_{r \theta}
\end{array}\right]
$$

where $\chi_{i j}$ are the stress components, $A_{i j}(i, j=r, \theta, z)$ and $D, E, F$ are elastic constant and $\nu_{i j}$ are strain components. Now for propagation of torsional surface wave in radial direction and causing displacement in azimuthal direction only, we have considered following components of displacement:

$$
w_{r}^{(1)}=0, \quad w_{\theta}^{(1)}=w_{\theta}^{(1)}(r, z, t), \quad w_{z}^{(1)}=0 .
$$

Using eq. (9), the strain-displacements relation for upper half-space are obtained as:

$$
\begin{aligned}
& \nu_{r r}=0, \quad \nu_{\theta \theta}=0, \quad \nu_{z z}=0, \\
& \nu_{\theta z}=\frac{1}{2} \frac{\partial w_{\theta}^{(1)}}{\partial z}, \quad \nu_{r z}=0, \quad \nu_{r \theta}=\frac{1}{2}\left(\frac{\partial w_{\theta}^{(1)}}{\partial r}-\frac{w_{\theta}^{(1)}}{r}\right) .
\end{aligned}
$$

Using eq. (10), in eq. (8), we have non-vanishing stress components are obtained as:

(11) $\chi_{r r}=\chi_{\theta \theta}=\chi_{z z}, \quad \chi_{r z}=0, \quad \chi_{\theta z}=D \frac{\partial w_{\theta}^{(1)}}{\partial z}, \quad \chi_{r \theta}=F\left(\frac{\partial w_{\theta}^{(1)}}{\partial z}-\frac{w_{\theta}^{(1)}}{r}\right)$, 
Torsional Wave Propagation in a Pre-Stressed Structure with Corrugated and ... 55

where $D$ and $F$ are rigidity of the medium along radial and axial direction, respectively.

Using eq. (11) in eq. (7), we have only the non-vanishing equation of motion for upper orthotropic half-space with initial stress is given by:

$$
F\left(\frac{\partial^{2} w_{\theta}^{(1)}}{\partial r^{2}}+\frac{1}{r} \frac{\partial w_{\theta}^{(1)}}{\partial r}-\frac{w_{\theta}^{(1)}}{r^{2}}\right)+\left(D-\frac{P}{2}\right) \frac{\partial^{2} w_{\theta}^{(1)}}{\partial z^{2}}=\rho_{1} \frac{\partial^{2} w_{\theta}^{(1)}}{\partial t^{2}}
$$

where $\beta_{1}=\sqrt{\frac{D}{\rho_{1}}}$ is the shear wave velocity for the upper layer.

Now the solution of eq. (12) is assumed as:

$$
w_{\theta}^{(1)}=W_{1}(z) J_{1}(k r) e^{i \omega t} .
$$

Using eq. (13) in eq. (12) we have:

$$
W_{1}^{\prime \prime}(z)+\phi^{2} W_{1}(z)=0,
$$

where

$$
\varphi^{2}=\left\{F\left(\frac{k^{2} J_{1}^{\prime \prime}(k r)}{\left(D-\frac{P}{2}\right) J_{1}(k r)}+\frac{k J_{1}^{\prime}(k r)}{r\left(D-\frac{P}{2}\right) J_{1}(k r)}-\frac{1}{r^{2}\left(D-\frac{P}{2}\right)}\right)+\frac{\rho_{1} \omega^{2}}{\left(D-\frac{P}{2}\right)}\right\} .
$$

Hence solution of eq. (14) is given by:

$$
W_{1}(z)=\left(A_{1} e^{i \phi z}+B_{1} e^{-i \phi z}\right)
$$

where $A_{1}$ and $B_{1}$ are arbitrary constants.

The appropriate solution of eq. (16) in view of condition:

$$
W_{1}(z) \rightarrow 0 \quad \text { when } \quad z \rightarrow \infty
$$

is:

$$
W_{1}(z)=A_{1} e^{i \phi z} .
$$

Now using eq. (18), eq. (13) results in:

$$
w_{\theta}^{(1)}=A_{1} e^{i \phi z} J_{1}(k r) e^{i \omega t} .
$$

Eq. (19) is the displacement for upper orthotropic half-space with initial stress.

\section{GOVERNING EQUATIONS AND SOLUTION FOR THE INTERMEDIATE LAYER}

Neglecting the viscosity of the liquid and body force, Biot's dynamical equations for porous layer under initial stress $P_{1}$ are given by (Biot [25]): 
(20) $\frac{\partial \varpi_{r r}}{\partial r}+\frac{1}{r} \frac{\partial \varpi_{r \theta}}{\partial r}+\frac{\partial \varpi_{r z}}{\partial z}+\frac{\varpi_{r r}-\varpi_{\theta \theta}}{r}$

$$
-P_{1}\left(\frac{\partial u_{z}}{\partial \theta}-\frac{\partial u_{\theta}}{\partial z}\right)=\frac{\partial^{2}}{\partial t^{2}}\left(\rho_{r r} w_{r}^{(2)}+\rho_{r \theta} W_{r}\right),
$$

(21) $\frac{\partial \varpi_{r \theta}}{\partial r}+\frac{2}{r} \frac{\partial \varpi_{r \theta}}{\partial r}+\frac{\partial \varpi_{\theta \theta}}{\partial \theta}+\frac{\partial \varpi_{\theta z}}{\partial z}-P_{1} \frac{\partial u_{z}}{\partial r}=\frac{\partial^{2}}{\partial t^{2}}\left(\rho_{r r} w_{\theta}^{(2)}+\rho_{r \theta} W_{\theta}\right)$,

(22) $\frac{\partial \varpi_{r z}}{\partial r}+\frac{1}{r} \frac{\partial \varpi_{z \theta}}{\partial \theta}+\frac{\partial \varpi_{z z}}{\partial z}+\frac{\varpi_{r z}}{r}+P_{1} \frac{\partial u_{\theta}}{\partial r}=\frac{\partial^{2}}{\partial t^{2}}\left(\rho_{r r} w_{z}^{(2)}+\rho_{r \theta} W_{z}\right)$,

$$
\frac{\partial \varpi}{\partial r}=\frac{\partial^{2}}{\partial t^{2}}\left(\rho_{r \theta} w_{r}^{(2)}+\rho_{\theta \theta} W_{r}\right), \quad \frac{\partial \varpi}{\partial \theta}=\frac{\partial^{2}}{\partial t^{2}}\left(\rho_{r \theta} w_{\theta}^{(2)}+\rho_{\theta \theta} W_{\theta}\right),
$$

(23) $\frac{\partial \varpi}{\partial z}=\frac{\partial^{2}}{\partial t^{2}}\left(\rho_{r \theta} w_{z}^{(2)}+\rho_{\theta \theta} W_{z}\right)$,

where $\varpi_{i j}$ are the components of stresses, $P_{1}$ is the initial stress and $\left(w_{r}^{(2)}, w_{\theta}^{(2)}\right.$, $\left.w_{z}^{(2)}\right),\left(W_{r}, W_{\theta}, W_{z}\right)$ are the components of solid phase displacements and fluid phase displacements, respectively.

The rotational components are:

$$
\begin{aligned}
& u_{r}=\frac{1}{2 r}\left(\frac{\partial w_{r}^{(2)}}{\partial \theta}-\frac{\partial w_{r}^{(2)}}{\partial z}\right), \\
& u_{\theta}=\frac{1}{2}\left(\frac{\partial w_{r}^{(2)}}{\partial z}-\frac{\partial w_{z}^{(2)}}{\partial r}\right), \\
& u_{z}=\frac{1}{2 r}\left(\frac{\partial}{\partial z} r w_{r}^{(2)}-\frac{\partial w_{r}^{(2)}}{\partial \theta}\right) .
\end{aligned}
$$

The relation between stress and displacement component are given as:

$$
\begin{aligned}
\alpha_{r r} & =\frac{1}{2}\left(\frac{\partial w_{r}^{(2)}}{\partial r}\right), \\
\alpha_{r \theta} & =\frac{1}{2}\left(\frac{1}{r} \frac{\partial w_{r}^{(2)}}{\partial \theta}+\frac{\partial w_{\theta}^{(2)}}{\partial r}-\frac{w_{\theta}^{(2)}}{r}\right), \\
\alpha_{\theta \theta} & =\frac{1}{2}\left(\frac{1}{r} \frac{\partial w_{\theta}^{(2)}}{\partial \theta}+\frac{w_{r}^{(2)}}{r}\right), \\
\alpha_{\theta z} & =\frac{1}{2}\left(\frac{1}{r} \frac{\partial w_{z}^{(2)}}{\partial \theta}+\frac{\partial w_{\theta}^{(2)}}{\partial z}\right) \\
\alpha_{r z}= & \frac{1}{2}\left(\frac{\partial w_{z}^{(2)}}{\partial r}+\frac{\partial w_{r}^{(2)}}{\partial z}\right), \\
\alpha_{z z}= & \frac{1}{2}\left(\frac{\partial w_{z}^{(2)}}{\partial z}\right) .
\end{aligned}
$$


Torsional Wave Propagation in a Pre-Stressed Structure with Corrugated and ... 57

The stress acting on the fluid phase may be represented as:

$$
q=-\pi p_{f},
$$

where $p_{f}$ is the pressure in the fluid and $\pi$ is the porosity of poroelastic layer. The mass coefficients $\rho_{r r}, \rho_{r \theta}, \rho_{\theta \theta}$ are related to total mass density of the solid-liquid aggregate $\left(\rho^{(2)}\right)$ and mass densities $p_{s}, p_{f}$ for solid and liquid, respectively by:

$$
\rho_{r r}+\rho_{r \theta}=(1-\varphi) p_{s}, \quad \rho_{r \theta}+\rho_{\theta \theta}=\varphi p_{f}, \quad \rho^{(2)}=\rho_{s}+\varphi\left(p_{f}-p_{s}\right),
$$

eq. (28) shows that as the porosity factor decreases from 1 to 0 , i.e., as the volume of pores decreases, the density of the aggregate tends to the density of the solid. It has been shown by Biot that the mass coefficients obey the following inequalities:

$$
\rho_{r r}>0, \quad \rho_{\theta \theta}>0, \quad \rho_{r r} \rho_{\theta \theta}-\rho_{r \theta^{2}}>0 .
$$

For the propagation of torsional surface wave along the radial direction we consider the displacement component in the following form:

$$
\begin{array}{ll}
w_{r}^{(2)}=w_{z}^{(2)}=0, & w_{\theta}^{(2)}=w_{\theta}^{(2)}(r, z, t), \\
W_{r}=W_{z}=0, & W_{\theta}=W_{\theta}(r, z, t) .
\end{array}
$$

In view of eq. (31) non-vanishing strain and rotational components are:

$$
\begin{array}{lll}
\alpha_{r \theta}=\frac{1}{2}\left(\frac{\partial w_{\theta}^{(2)}}{\partial r}-\frac{w_{\theta}^{(2)}}{r}\right), & \alpha_{\theta z}=\frac{1}{2}\left(\frac{\partial w_{\theta}^{(2)}}{\partial z}\right), \\
u_{r}=\frac{1}{2 r}\left(\frac{\partial w_{\theta}^{(2)}}{\partial z}\right), & u_{z}=\frac{1}{2 r} \frac{\partial}{\partial r}\left(r w_{\theta}^{(2)}\right) .
\end{array}
$$

Therefore non-vanishing stresses is given by:

$$
\varpi_{r \theta}=N^{\prime}\left(\frac{\partial w_{\theta}^{(2)}}{\partial r}-\frac{w_{\theta}^{(2)}}{r}\right), \quad \varpi_{z \theta}=L \frac{\partial w_{\theta}^{(2)}}{\partial z},
$$

where $N^{\prime}$ and $L$ are rigidity of the medium along radial and axial direction, respectively. Using eqs. (30), (31), (32), (33) and (34) in eqs. (20), (21), (22) and (23), we get:

$$
\left(N^{\prime}-\frac{P_{1}}{2}\right)\left(\frac{\partial^{2} w_{\theta}^{(2)}}{\partial r^{2}}+\frac{1}{r} \frac{\partial w_{\theta}^{(2)}}{\partial r}-\frac{w_{\theta}^{(2)}}{r^{2}}\right)+L \frac{\partial^{2} w_{\theta}^{(2)}}{\partial z^{2}}=\frac{\partial^{2}}{\partial t^{2}}\left(\rho_{r r} w_{\theta}^{(2)}+\rho_{r \theta} W_{\theta}\right)
$$




$$
\frac{\partial^{2}}{\partial t^{2}}\left(\rho_{r r} w_{\theta}^{(2)}+\rho_{r \theta} W_{\theta}\right)=0 .
$$

Eliminating $W_{\theta}$ from eqs. (35) and (36), we get:

(37) $\left(\frac{\partial^{2} w_{\theta}^{(2)}}{\partial r^{2}}+\frac{1}{r} \frac{\partial w_{\theta}^{(2)}}{\partial r}-\frac{w_{\theta}^{(2)}}{r^{2}}\right)+\frac{L}{\left(N^{\prime}-\frac{P_{1}}{2}\right)} \frac{\partial^{2} w_{\theta}^{(2)}}{\partial z^{2}}=\frac{P_{2}}{\left(N^{\prime}-\frac{P_{1}}{2}\right)} \frac{\partial^{2} w_{\theta}^{(2)}}{\partial t^{2}}$,

where

$$
P_{2}=\left(\rho_{r r}-\frac{\rho_{r \theta^{2}}}{\rho_{\theta \theta}}\right) .
$$

The solution of eq. (37) is assumed as:

$$
W_{\theta}^{(2)}=W_{2}(z) J_{1}(k r) e^{i \omega t} .
$$

Therefore using eq. (39) in eq. (37), then we obtain:

$$
\frac{d^{2} W_{2}}{d z^{2}}+\delta^{2} W_{2}=0
$$

where

$$
\delta^{2}=\frac{k^{2} N^{\prime}\left(1-\frac{P_{1}}{2 N^{\prime}}\right)}{L}\left\{\frac{Q}{N^{\prime}\left(1-\frac{P_{1}}{2 N^{\prime}}\right)} \frac{c^{2}}{\beta_{2}^{2}}-1\right\}, \quad Q=\frac{P_{2}}{\rho^{(2)}},
$$

$\beta_{2}=\sqrt{\frac{N^{\prime}}{\rho^{(2)}}}$ is the shear wave velocity for the porous layer.

The solution of eq. (40) is obtained as:

$$
V_{2}(z)=\left(A_{2} \sin \delta z+B_{2} \cos \delta z\right),
$$

where $A_{2}$ and $B_{2}$ are arbitrary constants. Therefore, expression of displacement in sandwiched poroelastic layer with corrugated boundary surfaces is given by:

$$
w_{\theta}^{(2)}=\left(A_{2} \sin \delta z+B_{2} \cos \delta z\right) J_{1}(k r) e^{i \omega t} .
$$




\section{GOVERNING EQUATIONS AND SOLUTION FOR THE LOWER HALF-SPACE}

The lower medium is considered non-homogeneous elastic half-space, hence nonvanishing equation of motion for propagation of torsional surface wave in pre-stressed heterogeneous anisotropic half-space given by:

$$
\frac{\partial \tau_{r \theta}}{\partial r}+\frac{\partial \tau_{\theta z}}{\partial z}+\frac{2}{r} \tau_{r \theta}-\frac{\partial}{\partial z}\left(\frac{P^{\prime \prime}}{2} \frac{\partial w_{\theta}^{(3)}}{\partial z}\right)=\rho_{3} \frac{\partial^{2} w_{\theta}^{(3)}}{\partial t^{2}},
$$

where $\rho_{3}$ is the density of the lower medium, $P^{\prime \prime}$ is the initial stress acting along radial direction in the medium and $\tau_{i j}$ is the component of stress in lower elastic half-space. Stress-displacement relations for non-vanishing stresses in heterogeneous anisotropic half-space are as:

$$
\begin{aligned}
\tau_{\theta z} & =L^{\prime} \frac{\partial w_{\theta}^{(3)}}{\partial z}, \\
\tau_{r \theta} & =A\left(\frac{\partial w_{\theta}^{(3)}}{\partial z}-\frac{w_{\theta}^{(3)}}{r}\right),
\end{aligned}
$$

where $L^{\prime}$ and $A$ are rigidity along radial and axial direction. Now the heterogeneity in the lower anisotropic half-space is assumed to be:

$A=A_{0} \cosh ^{2} \alpha z, \quad L^{\prime}=L_{0} \cosh ^{2} \alpha z, \quad \rho_{3}=\rho_{0} \cosh ^{2} \alpha z, \quad P^{\prime \prime}=P_{0} \cosh ^{2} \alpha z$.

Now using eqs. (45), (46) in eq. (44), we get:

$$
A\left(\frac{\partial^{2} w_{\theta}^{(3)}}{\partial r^{2}}+\frac{1}{r} \frac{\partial w_{\theta}^{(3)}}{\partial r}-\frac{w_{\theta}^{(3)}}{r^{2}}\right)+H \frac{\partial^{2} w_{\theta}^{(3)}}{\partial z^{2}}=\rho_{3} \frac{\partial^{2} w_{\theta}^{(3)}}{\partial t^{2}},
$$

where $H=\left(L^{\prime}-\frac{P^{\prime \prime}}{2}\right)$.

Using dimension-less coordinates $R=k r$ and $\gamma=z k+\gamma_{0}$, where $\gamma_{0}$ is the constants and $k$ is the wave number, then in eq. (48) we have:

(49) $\left(\frac{\partial^{2} w_{\theta}}{\partial R^{2}}+\frac{1}{R} \frac{\partial w_{\theta}^{(3)}}{\partial R}-\frac{w_{\theta}^{(3)}}{R^{2}}\right)+\frac{1}{A}\left(\frac{d H}{d \gamma} \frac{\partial w_{\theta}^{(3)}}{\partial \gamma}+H \frac{\partial^{2} w_{\theta}^{(3)}}{\partial \gamma^{2}}\right)=\frac{\rho_{3}}{A k^{2}} \frac{\partial^{2} w_{\theta}^{(3)}}{\partial t^{2}}$.

For the propagation of torsional surface wave propagating along the radial direction, we may assume the solution as:

$$
w_{\theta}^{(3)}=W_{3}(\gamma) w_{3}(r) e^{i \omega t} .
$$

Using eq. (50) in eq. (49), we have: 
(51) $\left\{\frac{d^{2} w_{3}}{d R^{2}}+\frac{1}{R} \frac{d w_{3}}{d R}+\left(1-\frac{1}{R^{2}}\right) w_{3}\right\} W_{3}$

$$
+\frac{H}{A}\left(\frac{d^{2} W_{3}}{d \gamma^{2}}+\frac{1}{H} \frac{d H}{d \gamma} \frac{d W_{3}}{d \gamma}+\frac{\left(\rho_{3} \omega^{2}-A k^{2}\right)}{H k^{2}} W_{3}\right) w_{3}=0 .
$$

The Equation:

$$
\frac{d^{2} w_{3}}{d R^{2}}+\frac{1}{R} \frac{d w_{3}}{d R}+\left(1-\frac{1}{R^{2}}\right) w_{3}=0,
$$

is the Bessel's Equation of first kind with solution $w_{3}=J_{1}(r)$ then above eq. (51) takes the form:

$$
\frac{d^{2} W_{3}}{d \gamma^{2}}+\frac{1}{H} \frac{d H}{d \gamma} \frac{d W_{3}}{d \gamma}+\frac{\left(\rho_{3} \omega^{2}-A k^{2}\right)}{H k^{2}} W_{3}=0
$$

Assuming $W_{3}=\frac{W}{\sqrt{H}}$ then eq. (53) can be written as:

$$
\frac{d^{2} W}{d \gamma^{2}}-\left\{\frac{1}{2 H} \frac{d^{2} H}{d \gamma^{2}}-\left(\frac{1}{2 H} \frac{d H}{d \gamma}\right)^{2}+\frac{A}{H}\left(1-\frac{\rho_{3} c}{A}\right) W\right\}=0 .
$$

Using eq. (47) in eq. (51), it takes the form:

$$
\frac{d^{2} W}{d \varpi^{2}}-\lambda_{3}^{2} W=0
$$

where:

$$
\lambda_{3}^{2}=\frac{\alpha^{2}}{k^{2}}+\frac{A_{0}}{L_{0}\left(1-\gamma_{0}\right)}\left(1-\frac{c^{2}}{\beta_{3}^{2}}\right)
$$

$$
\begin{aligned}
& \beta_{3}=\sqrt{\frac{A_{0}}{\rho_{0}}} \text { is the shear wave velocity of the lower half-space; } \\
& \gamma_{0}=\frac{P_{0}}{2 L_{0}} \text { is the non-dimensional initial stress parameter. }
\end{aligned}
$$

Solution of eq. (55) is given by:

$$
W(\gamma)=A_{3} e^{-\lambda_{3} \gamma}+B_{3} e^{\lambda_{3} \gamma},
$$

where $A_{3}$ and $B_{3}$ are arbitrary constants. 
Torsional Wave Propagation in a Pre-Stressed Structure with Corrugated and ... 61

The appropriate solution of eq. (57), in view of condition $W(\gamma) \rightarrow 0$ when $\gamma \rightarrow \infty$, is given by:

$$
W(\gamma)=A_{3} e^{-\lambda_{3} \gamma}
$$

Therefore, expression of displacement for lower heterogeneous corrugated prestressed anisotropic half-space is given by:

$$
w_{\theta}^{(3)}=\frac{A_{3} e^{-\lambda_{3} \gamma}}{\sqrt{H_{0}} \cosh \left(\frac{\alpha}{k}\right)\left(\gamma-\gamma_{0}\right)} J_{1}(R) e^{i \omega t},
$$

where:

$$
H_{0}=\left(L_{0}-\frac{P_{0}}{2}\right) \text {. }
$$

\section{BOUNDARY CONDITIONS AND DISPERSION RELATION}

The boundary conditions for the present problem are given as:

Stresses of heterogeneous orthotropic half-space and initial stressed poroelastic layer are equal at $z=g_{2}(r)-H$, which leads the following relation:

$$
\begin{aligned}
& \chi_{\theta z}-g_{2}^{\prime}(r) \chi_{r \theta}=\varpi_{\theta z}-g_{2}^{\prime}(r) \gamma_{r \theta}, \\
& \chi_{\theta z}-g_{2}^{\prime}(r) \chi_{r \theta}\left(1-\Omega_{1}\right)=i k N^{\prime} \Omega_{1} \frac{c}{\beta_{2}}\left(w_{\theta}^{(1)}-w_{\theta}^{(2)}\right),
\end{aligned}
$$

where $\Omega_{1}$ is bonding parameter of common interface of orthotropic half-space and initially stressed poroelastic layer. Stresses of initial stressed poroelastic layer and pre-stressed heterogeneous anisotropic half-space are equal, i.e. $z=g_{1}(r)$, which leads the following relation:

$$
\begin{aligned}
& \gamma_{\theta z}-g_{1}^{\prime}(r) \gamma_{r \theta}=\tau_{\theta z}-g_{1}^{\prime}(r) \tau_{r \theta}, \\
& \varpi_{\theta z}-g_{1}^{\prime}(r) \varpi_{r \theta}\left(1-\Omega_{2}\right)=i k A \Omega_{2} \frac{c}{\beta_{3}}\left(w_{\theta}^{(2)}-w_{\theta}^{(3)}\right),
\end{aligned}
$$

where $\Omega_{2}$ bonding parameter of common interface of initially stressed poroelastic layer and initially stressed heterogeneous anisotropic half-space.

On applying (i) boundary condition, we get:

$$
A_{1} M \varphi e^{i \varphi\left[g_{1}(r)-H\right]}=L \delta\left[A_{2} \cos \left(g_{1}(r)-H\right)-B_{2} \sin \left(g_{1}(r)-H\right)\right] .
$$

On applying (ii) boundary condition, we obtain: 
(66)

$$
\begin{aligned}
A_{1} M \varphi e^{i \varphi\left[g_{1}(r)-H\right]}\left(1-\Omega_{1}\right)= & i k N^{\prime} \Omega_{1} \frac{c}{\beta_{2}}\left[A_{1} e^{i \varphi\left[g_{1}(r)-H\right]}\right. \\
& \left.-A_{2} \sin \left(g_{1}(r)-H\right)-B_{2} \cos \left(g_{1}(r)-H\right)\right] .
\end{aligned}
$$

On applying (iii) boundary condition, we result in:

$$
\begin{aligned}
& L \delta\left[A_{2} \cos g_{2}(r)-B_{2} \sin g_{2}(r)\right] \\
& \quad=-L^{\prime} A_{3} e^{-\lambda_{3}\left(\gamma_{0}+g_{2}(r) k\right)}\left(\frac{-\lambda_{3} k \cosh \alpha g_{2}(r)+\alpha \sinh g_{2}(r)}{\sqrt{H_{0}} \cos ^{2} h \alpha g_{2}(r)}\right) .
\end{aligned}
$$

On applying (iii) boundary condition, we have:

$$
\begin{aligned}
& L \delta\left[A_{2} \cos g_{2}(r)-B_{2} \sin g_{2}(r)\right]\left(1-\Omega_{2}\right)= \\
& \quad i k A \Omega_{2} \frac{c}{\beta_{3}}\left(A_{2} \sin g_{2}(r)+B_{2} \cos g_{2}(r)\right)-A_{3} \frac{e^{-\lambda_{3}\left(\gamma_{0}+g_{2}(r) k\right)}}{\sqrt{H_{0}} \cosh \alpha g_{2}(r)} .
\end{aligned}
$$

From eq. (67) and eq. (68), eliminating $A_{3}$ we have:

$$
\begin{aligned}
& A_{2}\left\{\left[L \delta\left(1-\Omega_{2}\right) \cos \delta g_{2}(r)-i k A \Omega_{2} \frac{c}{\beta_{3}} \sin \delta g_{2}(r)\right] \cosh \alpha g_{2}(r)\right. \\
& \left.\quad-i k A \Omega_{2} \frac{c}{\beta_{3}}\left(\frac{L \delta \cos \delta g_{2}(r) \cos ^{2} h \alpha g_{2}(r)}{L^{\prime}\left(-\lambda_{3} k \cosh \alpha g_{2}(r)+\alpha \sinh \alpha g_{2}(r)\right)}\right)\right\} \\
& -B_{2}\left\{\left[L \delta\left(1-\Omega_{2}\right) \sin \delta g_{2}(r)+i k A \Omega_{2} \frac{c}{\beta_{3}} \cos \delta g_{2}(r)\right] \cosh \alpha g_{2}(r)\right. \\
& \left.\quad+i k A \Omega_{2} \frac{c}{\beta_{3}}\left(\frac{L \delta \sin \delta g_{2}(r) \cos ^{2} h \alpha g_{2}(r)}{L^{\prime}\left(-\lambda_{3} k \cosh \alpha g_{2}(r)+\alpha \sinh \alpha g_{2}(r)\right)}\right)\right\}=0 .
\end{aligned}
$$

Eliminating constants from eq. (65), (66) and (69) and applying the necessary and sufficient conditions for the homogeneous algebraic equation, we get dispersion equation for the torsional wave in following form:

$$
\text { (70) } \begin{aligned}
M \varphi & {\left[\sin \delta\left(g_{1}(r)-H\right)\left(L \delta\left(1-\Omega_{2}\right) \sin \delta g_{2}(r)+i k A \Omega_{2} \frac{c}{\beta_{3}} \cos \delta g_{2}(r)\right)\right] \cosh \alpha g_{2}(r) } \\
& +\left[i k A \Omega_{2} \frac{c}{\beta_{3}}\left(\frac{L \delta \sin \delta g_{2}(r) \cos ^{2} h \alpha g_{2}(r)}{L^{\prime}\left(-\lambda_{3} k \cosh \alpha g_{2}(r)+\alpha \sinh \alpha g_{2}(r)\right)}\right)\right] \\
& -\left(L \delta\left(1-\Omega_{2}\right) \cos \delta g_{2}(r)-i k A \Omega_{2} \frac{c}{\beta_{3}} \sin \delta g_{2}(r)\right)\left[\cosh \alpha g_{2}(r)\right. \\
& \left.-i k A \Omega_{2} \frac{c}{\beta_{3}}\left(\frac{L \delta \cos \delta g_{2}(r) \cos ^{2} h \alpha g_{2}(r)}{L^{\prime}\left(-\lambda_{3} k \cosh \alpha g_{2}(r)+\alpha \sinh \alpha g_{2}(r)\right)}\right)\left\{\cos \delta\left(g_{1}(r)-H\right)\right\}\right]
\end{aligned}
$$




$$
\begin{aligned}
& -\left\{M \varphi\left(1-\Omega_{1}\right)-i k N^{\prime} \Omega_{1} \frac{c}{\beta_{2}}\right\}\left\{L \delta \cos \delta\left(g_{1}(r)-H\right)\right\} \\
& \times\left[L \delta\left(1-\Omega_{2}\right) \sin \delta g_{2}(r)+i k A \Omega_{2} \frac{c}{\beta_{3}} \cos \delta g_{2}(r)\right]\left[\cosh \alpha g_{2}(r)\right. \\
& \left.+i k A \Omega_{2} \frac{c}{\beta_{3}}\left(\frac{L \delta \sin \delta g_{2}(r) \cos ^{2} h \alpha g_{2}(r)}{L^{\prime}\left(-\lambda_{3} k \cosh \alpha g_{2}(r)+\alpha \sinh \alpha g_{2}(r)\right)}\right)\right] \\
& -\left\{L \delta \sin \delta\left(g_{1}(r)-H\right)\right\}\left[L \delta\left(1-\Omega_{2}\right) \cos \delta g_{2}(r)-i k A \Omega_{2} \frac{c}{\beta_{3}} \sin \delta g_{2}(r)\right] \cosh \alpha g_{2}(r) \\
& -i k A \Omega_{2} \frac{c}{\beta_{3}}\left(\frac{L \delta \cos \delta g_{2}(r) \cos ^{2} h \alpha g_{2}(r)}{L^{\prime}\left(-\lambda_{3} k \cosh \alpha g_{2}(r)+\alpha \sinh \alpha g_{2}(r)\right)}\right)=0 .
\end{aligned}
$$

For numerical calculation and graphical illustration of present study, we will take velocity Eq. (70) into our account with:

$$
g_{1}(r)=\kappa_{1} \cos (b r), \quad g_{2}(r)=\kappa_{2} \cos (b r),
$$

where $\kappa_{1}, \kappa_{2}$ are the flatness parameters of the layer and lower half-space, respectively.

\section{SPECIAL CASES}

Case 1: When upper interface and lower interface are corrugated and given by means of periodic functions $g_{1}(r)=\kappa_{1} \cos (b r), g_{2}(r)=\kappa_{2} \cos (b r)$ and both the interfaces are welded in contact (i.e., $\Omega_{1}, \Omega_{2} \rightarrow 1$ ) then velocity equation of torsional surface wave (70) reduces to

$$
\begin{aligned}
M \varphi & {\left[\sin \delta\left(\kappa_{1} \cos (b r)-H\right)\right]\left\{i k A \Omega_{2} \frac{c}{\beta_{3}} \cos \delta \kappa_{2} \cos (b r)\right\} \cosh \alpha \kappa_{2} \cos (b r) } \\
& +i k A \Omega_{2} \frac{c}{\beta_{3}}\left(\frac{L \delta \sin \delta \kappa_{2} \cos (b r) \cos ^{2} h \alpha \kappa_{2} \cos (b r)}{L^{\prime}\left(-\lambda_{3} k \cosh \alpha g_{2}(r)+\alpha \sinh \alpha g_{2}(r)\right)}\right) \\
& +i k A \Omega_{2} \frac{c}{\beta_{3}} \sin \delta \kappa_{2} \cos (b r) \cosh \alpha \kappa_{2} \cos (b r) \\
& -i k A \Omega_{2} \frac{c}{\beta_{3}}\left(\frac{L \delta \cos \delta \kappa_{2} \cos (b r) \cos ^{2} h \alpha \kappa_{2} \cos (b r)}{L^{\prime}\left(-\lambda_{3} k \cosh \alpha \kappa_{2} \cos (b r)+\alpha \sinh \alpha \kappa_{2} \cos (b r)\right)}\right) \\
& \times \cos \delta\left(\kappa_{1} \cos (b r)-H\right) i k N^{\prime} \Omega_{1} \frac{c}{\beta_{2}}\left\{L \delta \cos \delta\left(\kappa_{1} \cos (b r)-H\right)\right\} \\
& \times i k A \Omega_{2} \frac{c}{\beta_{3}} \cos \delta \kappa_{2} \cos (b r) \cosh \alpha \kappa_{2} \cos (b r) \\
& +i k A \Omega_{2} \frac{c}{\beta_{3}}\left(\frac{L \delta \sin \delta \kappa_{2} \cos (b r) \cos { }^{2} h \alpha \kappa_{2} \cos (b r)}{L^{\prime}\left(-\lambda_{3} k \cosh \alpha \kappa_{2} \cos (b r)+\alpha \sinh \alpha \kappa_{2} \cos (b r)\right)}\right) \\
& +\left\{L \delta \sin \delta\left(\kappa_{1} \cos (b r)-H\right)\right\} i k A \Omega_{2} \frac{c}{\beta_{3}} \sin \delta \kappa_{2} \cos (b r) \cosh \alpha \kappa_{2} \cos (b r) \\
& -i k A \Omega_{2} \frac{c}{\beta_{3}}\left(\frac{L \delta \cos \delta \kappa_{2} \cos (b r) \cos { }^{2} h \alpha \kappa_{2} \cos (b r)}{L^{\prime}\left(-\lambda_{3} k \cosh \alpha \kappa_{2} \cos (b r)+\alpha \sinh \alpha \kappa_{2} \cos (b r)\right)}\right)=0 .
\end{aligned}
$$

Equation (71) represents dispersion relation for torsional surface waves, when initially stressed porous layer with is sandwiched between orthotropic half-space 
with initial stress and prestressed heterogeneous anisotropic layer, having corrugated boundary surface and both the interfaces are welded in contact.

Case 2: When uppermost interface and lowermost interface are without corrugation (planner) (i.e., $g_{1}(r)=0$ and $\left.g_{1}(r)=0\right)$ and both interfaces are welded in contact (i.e., $\Omega_{1}, \Omega_{2} \rightarrow 1$ ), then velocity equation of torsional surface wave (70) reduces to:

$$
\begin{aligned}
-M \varphi \sin \delta H i k A \frac{c}{\beta_{3}}+i k A \frac{c}{\beta_{3}} \frac{L}{L^{\prime} \lambda_{3} k} & \cos \delta H \\
& +k^{2} A N^{\prime} \frac{c^{2}}{\beta_{2}^{2}} L^{2} \delta^{2} \cos \delta H \sin \delta H=0 .
\end{aligned}
$$

Equation (72) highlights the dispersion relation for torsional surface waves, when an initially stressed porous layer, having both the interfaces are planner and welded in contact sandwiched between orthotropic half-space under initial stress and prestressed heterogeneous anisotropic half-space.

Case 3: When uppermost interface and lowermost interface are without corrugation (planner) (i.e., $g_{1}(r)=0$ and $g_{1}(r)=0$ ) and both the interfaces are welded in contact (i.e., $\Omega_{1}, \Omega_{2} \rightarrow 1$ ), upper half-space, intermediate layer is without initial stress (i.e., $\left.P, P_{1}=0\right)$ and lower half-space is also without initial stress (i.e., $\left.P^{\prime \prime}=0\right)$ then velocity equation for torsional surface wave (70) reduces to

$$
\begin{aligned}
-M \varphi^{\prime} \sin \delta^{\prime} H i k^{\prime} A \frac{c}{\beta_{3}}+i k A \frac{c}{\beta_{3}} & \frac{L}{L^{\prime} \lambda_{3} k} \cos \delta^{\prime} H \\
& \quad+k^{2} A N^{\prime} \frac{c^{2}}{\beta_{2}^{2}} L^{2} \delta^{\prime 2} \cos \delta^{\prime} H \sin \delta H=0,
\end{aligned}
$$

where:

$$
\begin{aligned}
\delta^{\prime 2} & =k^{2}\left(\frac{N^{\prime}}{L}\left\{Q \frac{c^{2}}{\beta_{2}^{2}}-1\right\}\right) \text { and } \\
{\varphi^{\prime}}^{2} & =N\left(\frac{k^{2} J_{1}^{\prime \prime}(k r)}{M J_{1}(k r)}+\frac{k J_{1}^{\prime}(k r)}{r M J_{1}(k r)}-\frac{1}{r^{2} M}\right)+\frac{\rho_{1} \omega^{2}}{M} .
\end{aligned}
$$

Equation (73) highlights the dispersion relation for torsional surface waves, when an initial stress free porous layer, having both the interfaces are planer and welded in contact is sandwiched between orthotropic half-space free from initial stress and heterogeneous anisotropic half-space without initial stress.

Case 4: When uppermost interface and lowermost interface are without corrugation (planner) (i.e., $g_{1}(r)=0$ and $\left.g_{1}(r)=0\right)$ and both the interfaces are welded in contact 
(i.e., $\Omega_{1}, \Omega_{2} \rightarrow 1$ ), upper half-space, without initial stress and isotropic (i.e., $P=0$, $\left.M=N=\mu_{1}\right)$, intermediate layer is without initial stress and non-porous isotropic elastic (i.e., $Q \rightarrow 1, N^{\prime}=L=\mu_{2}, P^{\prime}=0$ ) and lower half-space is also without initial stress, homogeneous and isotropic (i.e., $P^{\prime \prime}=0, \alpha=0, P_{0}=L_{0}=\mu$, $\gamma_{0} \rightarrow 0$ ), then velocity equation for torsional surface wave (70) reduces to:

$$
\begin{aligned}
\left(-M \varphi_{1} \sin \delta_{1} H i k A \frac{c}{\beta_{3}}+i k A\right. & \frac{c}{\beta_{3}} \frac{L}{L^{\prime} \lambda_{3} k} \cos \delta_{1} H \\
& \left.+k^{2} A N^{\prime} \frac{c^{2}}{\beta_{2}^{2}} L^{2} \delta_{1}^{2} \cos \delta_{1} H \sin \delta_{1} H\right)=0
\end{aligned}
$$

where:

$$
\begin{aligned}
& \delta_{1}^{2}=k^{2}\left(\mu_{2}\left\{\frac{c^{2}}{\beta_{2}^{2}}-1\right\}\right) \text { and } \\
& \varphi_{1}^{2}=\mu_{1}\left(\frac{k^{2} J_{1}^{\prime \prime}(k r)}{\mu_{1} J_{1}(k r)}+\frac{k J_{1}^{\prime}(k r)}{r \mu_{1} J_{1}(k r)}-\frac{1}{r^{2} \mu_{1}}\right)+\frac{\rho_{1} \omega^{2}}{\mu_{1}} .
\end{aligned}
$$

Equation (74) highlights the dispersion relation for torsional surface waves, when an initial stress non- porous, isotropic elastic having both interfaces are planner and welded in contact is sandwiched between isotropic orthotropic half-space free from initial stress and isotropic homogeneous half-space without initial stress.

Case 5: When upper half-space is absent, intermediate layer is without corrugation, initial stress and non-porous isotropic elastic (i.e., $Q \rightarrow 1, N^{\prime}=L=\mu_{2}, P^{\prime}=0$ ) and lower half-space is also without initial stress, homogeneous and isotropic (i.e., $\left.P^{\prime \prime}=0, \alpha=0, P_{0}=L_{0}=\mu, \gamma_{0} \rightarrow 0\right)$, then velocity equation for torsional surface wave (70) reduces to:

$$
\tan k H \sqrt{\frac{c^{2}}{\beta_{2}^{2}}-1}=\mu_{3} \sqrt{1-\frac{c^{2}}{\beta_{3}^{2}}} \mu_{2} / \sqrt{\frac{c^{2}}{\beta_{2}^{2}}-1},
$$

which is classical Love equation. Equation (75) describes the dispersion equation for the torsional surface wave, when a homogeneous layer is overlying an isotropic homogeneous half-space, which is validation of classical Love equation.

\section{NUMERICAL CALCULATION AND DISCUSSION}

For numerical illustration and graphical interpretation we have taken following data:

(1) For upper orthotropic half-space with initial stress $\left(M_{2}\right)$

(Prosser and Green [26])

$$
M=2.64 \times 10^{9} \mathrm{~N} / \mathrm{m}^{2}, \quad N=1.87 \times 10^{9} \mathrm{~N} / \mathrm{m}^{2}, \quad \rho_{1}=1442 \mathrm{~kg} / \mathrm{m}^{3} ;
$$


(2) For intermediate initially stressed porous layer $\left(M_{2}\right)$ (Batugin and Nirenburg [27])

$$
N^{\prime}=0.49 \times 10^{9} \mathrm{~N} / \mathrm{m}^{2}, \quad L=0.52 \times 10^{9} \mathrm{~N} / \mathrm{m}^{2} \text { and } \rho_{2}=2435 \mathrm{~kg} / \mathrm{m}^{3} ;
$$

(3) For pre-stressed inhomogeneous anisotropic half-space $\left(M_{3}\right)$ (Gubbins [28])

$$
L^{\prime}=3.88 \times 10^{8} \mathrm{~N} / \mathrm{m}^{2}, \quad A=5.82 \times 10^{8} \mathrm{~N} / \mathrm{m}^{2}, \quad \rho_{3}=4744 \mathrm{~kg} / \mathrm{m}^{3} .
$$

The variation of phase velocity $\left(c / \beta_{2}\right)$ of torsional surface wave against dimensionless wave number $(k H)$ for different value of various affecting parameter, as bonding parameter for upper $\left(\Omega_{1}\right)$ and lower $\left(\Omega_{2}\right)$ half-space, initial stress acting in upper half-space $(P)$, sandwiched layer $\left(P_{1}\right)$ and lower half-space $\left(P_{2}\right)$, undulatory parameter $(b)$, flatness parameter for upper half-space $\left(\kappa_{1}\right)$ and for lower half-space $\left(\kappa_{2}\right)$ and porosity parameter $(Q)$, acting in intermediate layer have been shown in Figs 2 to 11 .

Figures 2 and 3 analyze the effect of dimensionless phase velocity against dimensionless wave number for different value bonding parameter, acting in upper and

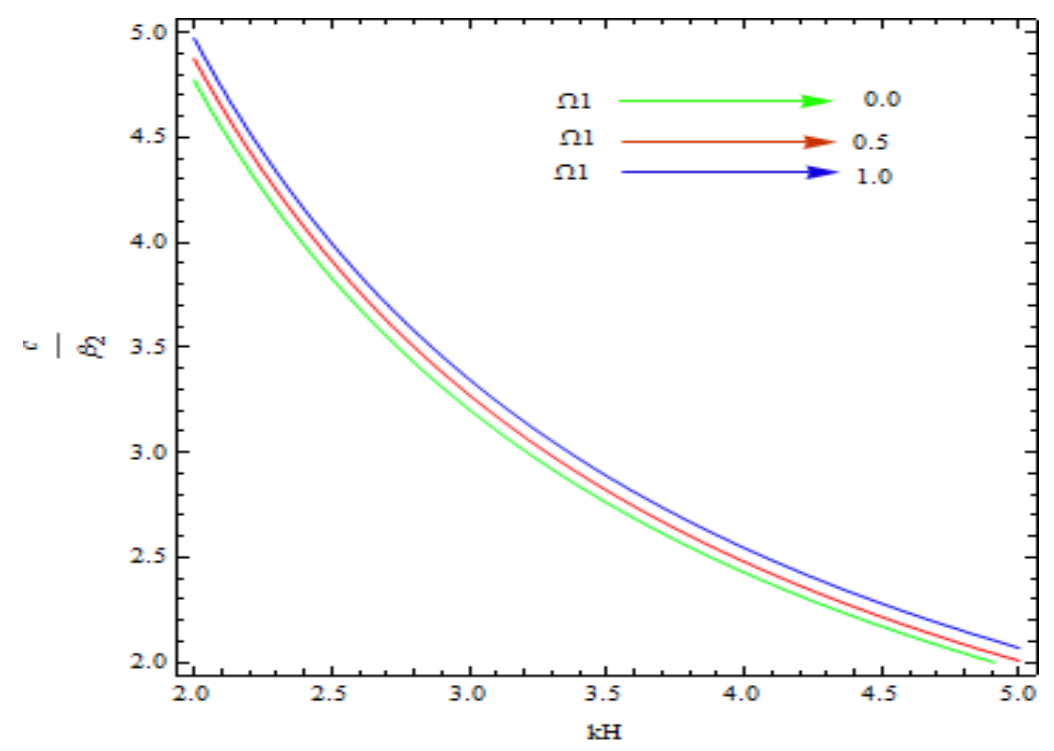

Fig. 2. Variation of dimensionless phase velocity $\left(c / \beta_{2}\right)$ against dimensionless wave number $(k H)$, for different values of upper bonding parameter $\left(\Omega_{1}\right)$, when $\Omega_{2}=1.4$. 


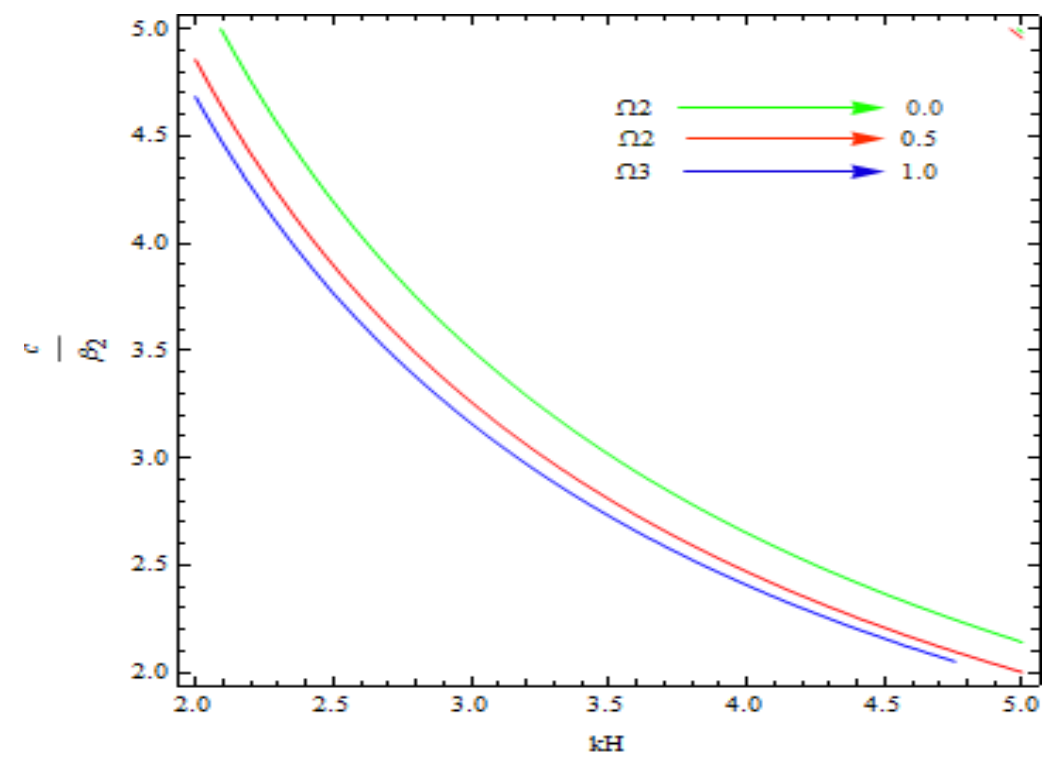

Fig. 3. Variation of dimensionless phase velocity $\left(c / \beta_{2}\right)$ against dimensionless wave number $(k H)$ for different values of lower bonding parameter $\left(\Omega_{2}\right)$, when $\Omega_{1}=1.3$.

lower half-space. In Figs 2 and 3 curves 1 represent the case when the medium is ideally smooth, while curve 2 related to the case, when the medium is loosely bonded and curve 3 is associated to the case, when the medium is perfectly bonded. Figure 2 explains that when bonding parameter acting in upper half-space increases, than the phase velocity of torsional surface wave increases. Figure 3 concludes that bonding parameter acting in lower half-space has reverse effect on the phase velocity of torsional surface wave.

Figures 4 and 5 highlight the effect of dimensionless phase velocity against dimensionless wave number for different value flatness parameter, acting in upper and lower half-space. In Figs 4 and 5 curves 1 represent the case when the medium is without corrugation, while curves 2 and 3 are related to the case when the medium is corrugated. Figure 4 shows that flatness parameter for the upper half-space has opposing behaviour on the phase velocity of torsional wave. Figure 5 explains that increasing value of lower flatness parameter increases the phase velocity of torsional surface waves.

Figure 6 examines the effect of corrugation parameter on the phase velocity of torsional wave. In Fig. 6 we can observe that corrugation parameter associated with corrugated boundary surfaces has adverse effect on the phase velocity of torsional surface waves. 


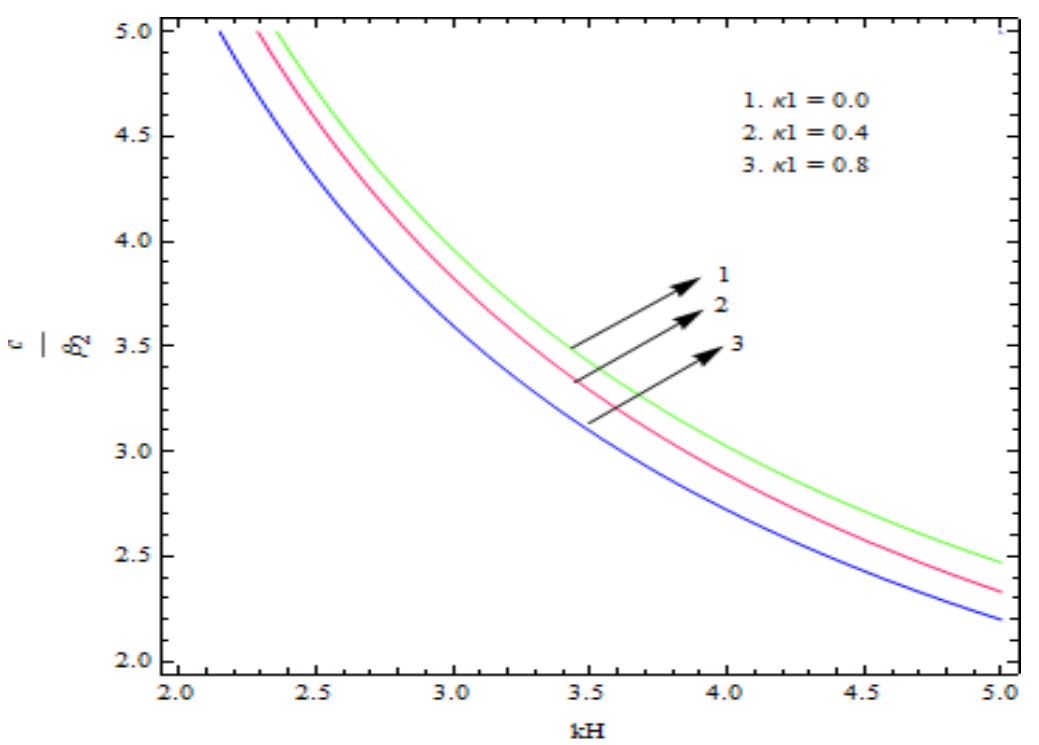

Fig. 4. Variation of dimensionless phase velocity $\left(c / \beta_{2}\right)$ against dimensionless wave number $(k H)$ for different values of upper flatness parameter $\left(\kappa_{1}\right)$ when $\kappa_{2}=0.3$.

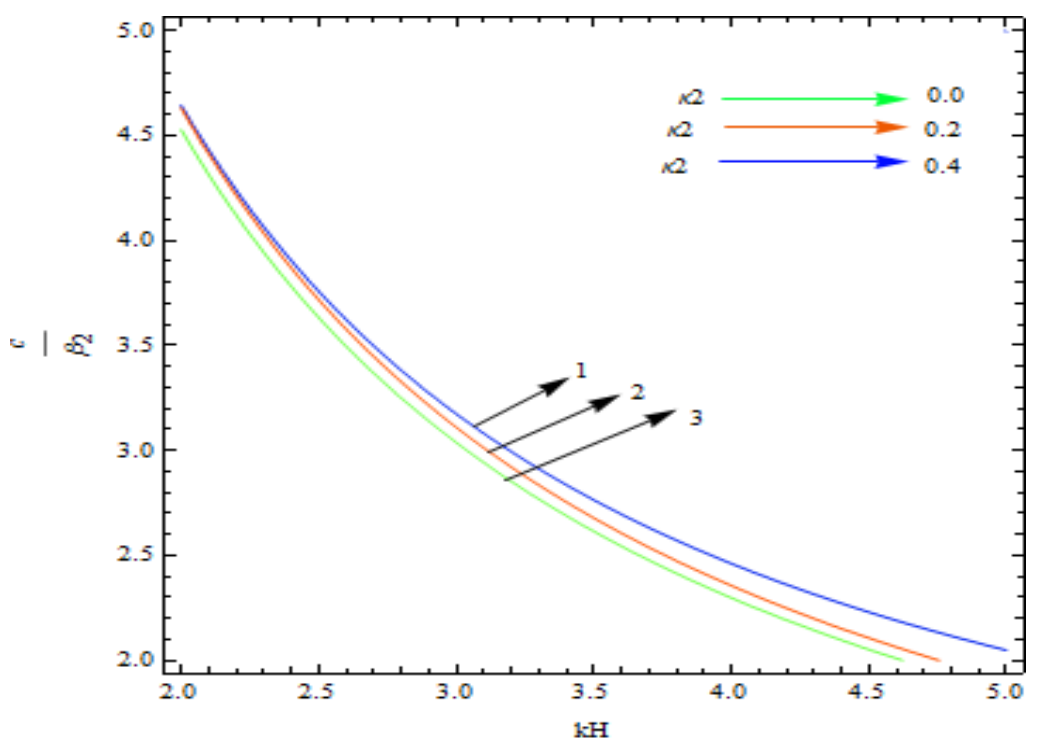

Fig. 5. Variation of dimensionless phase velocity $\left(c / \beta_{2}\right)$ against dimensionless wave number $(k H)$ for different values of upper flatness parameter $\left(\kappa_{2}\right)$ when $\kappa_{1}=0.3$. 
Torsional Wave Propagation in a Pre-Stressed Structure with Corrugated and ... 69

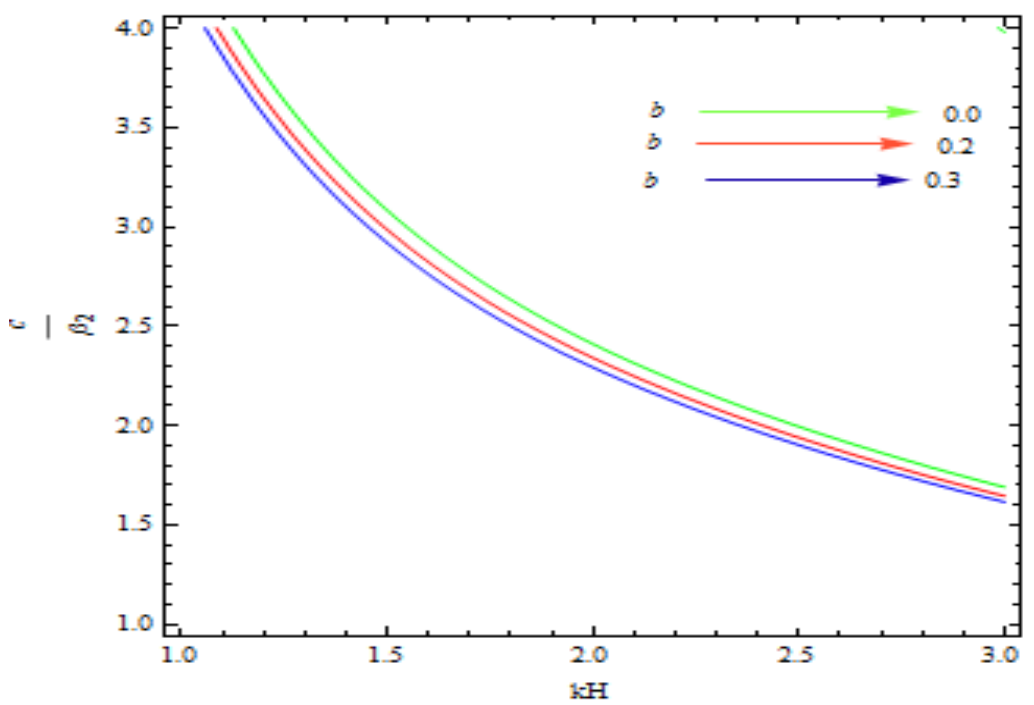

Fig. 6. Variation of dimensionless phase velocity $\left(c / \beta_{2}\right)$ against dimensionless wave number $(k H)$ for different values of undulation parameter $(b)$, when $\kappa_{1}=0.3$ and $\kappa_{2}=0.4$.

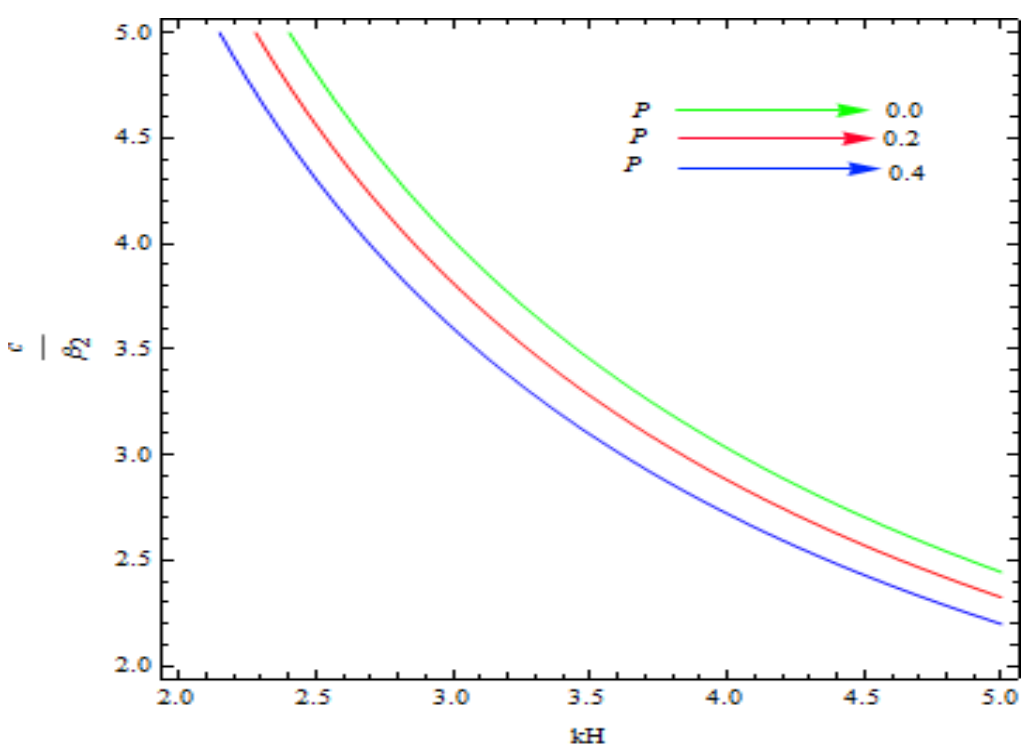

Fig. 7. Variation of dimensionless phase velocity $\left(c / \beta_{2}\right)$ against dimensionless wave number $(k H)$ for different values of upper initial stress $(P)$ when $P^{\prime} / 2 N^{\prime}=0.8$ and $P_{0} / 2 L_{0}=1.6$. 


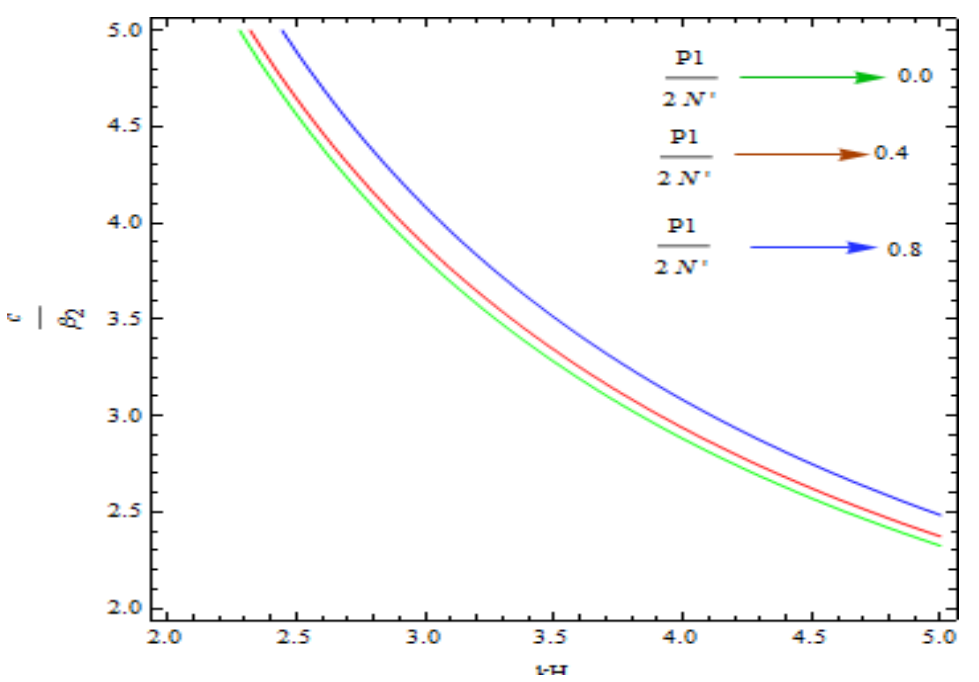

Fig. 8. Variation of dimensionless phase velocity $\left(c / \beta_{2}\right)$ against dimensionless wave number $(k H)$ for different values of intermediate initial stress $\left(P_{1} / 2 N^{\prime}\right)$ when $P=0.4$ and $P_{0} / 2 L_{0}=1.6$.

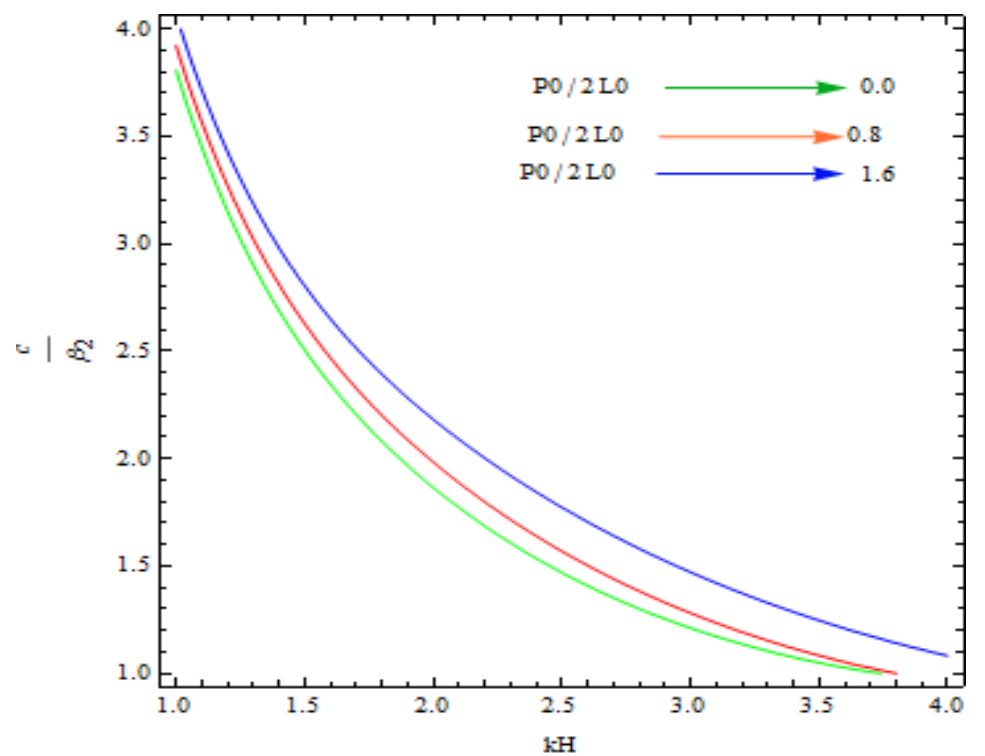

Fig. 9. Variation of dimensionless phase velocity $\left(c / \beta_{2}\right)$ against dimensionless wave number $(k H)$ for different values of lower initial stress $\left(P_{0} / 2 L_{0}\right)$, when $P^{\prime} / 2 N^{\prime}=0.8$ and $P=0.4$. 
Figures 7, 8 and 9 represent the effect of phase velocity of torsional wave against wave number for different values of initial stress acting in upper half-space intermediate layer and lower half-space, respectively. In each of the Figs 7, 8 and 9 first curve showing the case, when the corresponding boundary surface is free from initial stress, whereas curve 2 and 3 is related with the case corresponding boundary surface is with initial stress. It is observed from Figs 7 and 9 that initial stress acting in upper and lower half-space have reverse effect on the phase velocity of torsional surface waves. Fig. 8 exhibits that initial stress associated with intermediate layer has favouring effect on the phase velocity of torsional surface waves. A quick inspection on the Figs 7, 8 and 9 indicate that the initial stress acting in upper half-space, sandwiched porous layer and lower half-space have their individual significance, as they do not appear with the same effect.

Figure 10 shows the effect of porosity parameter $(Q)$ acting in intermediate poroelastic layer on the phase velocity of torsional wave. In Fig. 10 curve 1 manifest the case of boundary surface free from porosity and curve 2, 3 analyze the case when the porosity present in the medium. In Fig. 10 it can be highlighted that porosity parameter has adverse effect on the phase velocity of torsional wave.

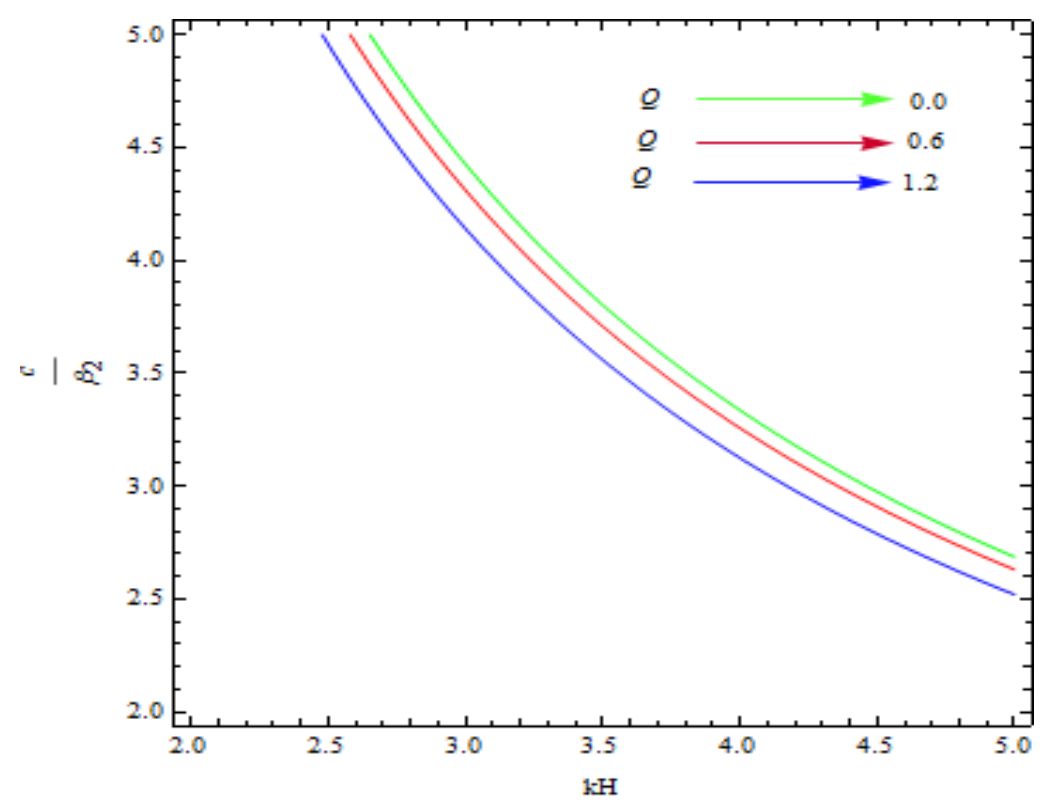

Fig. 10. Variation of dimensionless phase velocity $\left(c / \beta_{2}\right)$ against dimensionless wave number $(k H)$ for different values of porosity parameter $(Q)$. 


\section{CONCLUSION}

The current problem manifest the propagation of torsional wave in initially stressed porous layer, sandwiched between an orthotropic half-space with initial stress and prestrssed inhomogeneous anisotropic half-space. The effect of different parameter, e.g. porosity, heterogeneity, initial stress, flatness parameter, bonding parameter and undulation parameter have been analyzed. Major outcomes is obtained as follows:

1. The dispersion equation for the torsional surface waves, propagating in an initially stresses porous layer, sandwiched between orthotropic half-space with initial stress and pre-stressed inhomogeneous anisotropic half-space obtained in closed form.

2. The phase velocity of torsional surface wave decreases with increases in wave number in all the cases.

3. Bonding parameter for the upper half-space and initial stress associated with porous layer, flatness parameter acting in lower half-space, initial stress related to the lowermost inhomogeneous anisotropic half-space and intermediate porous layer have favouring effect on phase velocity of torsional surface waves.

4. Undulation parameter, flatness parameter associated with upper half-space, bonding parameter for the lower half-space, initial stress acting in uppermost orthotropic half-space and porosity parameter acting in intermediate poroelastic layer have adverse effect on the phase velocity of torsional wave.

5. In isotropic, homogeneous and porous-free case without initial stress, the dispersion relation of the problem matches with the classical Love-wave equation. This shows that the problem is in well agreement to the classical case (problem).

The velocity profile of surface waves are affected by the medium/ layers through which they travel. The Geological studies have established the fact, that in the large span of earth crust a series of rocks may be found over the water reservoirs, which are porous in nature. The results obtained from this study are likely to give better information about the porous layers saturated with oil and water in the crust and upper mantle. The results may be useful for exploration purposes. The considered model is motivated by such geological conditions within the earth. In present paper boundaries of the layers are considered to be non-planner (Corrugated) and loosely in order to make the problem statement close to the real situation. The current study is highly applicable in many projects, technological, scientific and engineering fields such as geophysics, seismology, civil engineering. The obtained results are useful and 
interesting and actual for researchers dealing with wave propagation in mechanical composite layered structures with complex mechanical properties.

\section{REFERENCES}

[1] Selim, M. M. Static Deformation of an Irregular Initially Stressed Medium. Appl. Math. Comput., 188 (2007), 1274-1284.

[2] ZHANG, R., M. SHINOZUKA. Effects of Irregular Boundaries in a Layered Half-space on Seismic Waves. Journal of Sound Vibration, 195 (1996), 1-16.

[3] Chen, X. F. Generation and Propagation of Seismic SH Waves in Multi-layered Media with Irregular Interfaces. Adv. Geophys., 48 (2007), 191-264.

[4] Singh, S. S., S. K. Tomar. qP-wave at a Corrugated Interface between Two Dissimilar Pre-stressed Elastic Half-spaces. J Sound Vib., 317 (2008), No. 3, 687-708.

[5] Singh, S. S. Love Wave at a Layer Medium Bounded by Irregular Boundary Surfaces. Journal of Vibration and Control, 17 (2011), 789-795.

[6] Rayleigh, L. On the Reflection of Sound or Light from a Corrugated Surface. Rep. Brit. Assoc. Adv. Sci., (1893), 690-691.

[7] Asano, S. Reflection and Refraction of Elastic Waves at a Corrugated Boundary Surface: Part I: The Case of Incidence of SH-wave. Bulletin of the Earthquake Research Institute, 38 (1960), No. 2, 177-197.

[8] Vardoulakis, I. Torsional Surface Waves in Inhomogeneous Elastic Media. Int. J. Numer. Anal. Methods Geomech., 8 (1984), No. 3, 287-296.

[9] Georgiadis, H. G., I. Vardoulakis, G. Lykotrafitis. Torsional Surface Waves in a Gradient-elastic Half-space. Wave Motion, 31 (2000), No. 4, 333-348.

[10] Bao, L., H. Yuan, M. Sakurai, M. Nakazawa, K. Kemmochi. A Study on the Torsional Wave of Fibre Reinforced Composite Materials. J. Compos. Mater, 40 (2006), No. 4, 338-391.

[11] Vishwakarma, S. K., S. Gupta. Torsional Surface Wave in a Homogeneous Crustal Layer over a Viscoelastic Mantle. Int. J. Appl. Math. Mech., 8 (2012), No. 16, 38-50.

[12] LiU, H., Z. K. WANG, T. J. WANG. Effect of Initial Stress on the Propagation Behaviour of Love Waves in a Layered Piezoelectric Structure. International Journal of Solids and Struct., 38 (2001), 37-51.

[13] Qian, J., F. Jin, Z. WANG, K. Kishimoto. Love Wave Propagation in a Piezoelectric Layered Structure with Initial Stresses. Acta Mech., 171 (2004), No. 1, 41-47.

[14] Biot, M. A. The Influence of Initial Stress on Elastic Waves. J. Appl. Phys., 11 (1940), 522-530.

[15] Dey, S., M. Chakraborty. Influence of Gravity and Ynitial Stress on the Love Waves in a Transversely Isotropic Medium. Geophys. Res. Bull., 21 (1983), 311-323.

[16] Akbarov, S., N. Ilhan. Dynamics of a System Comprising a Pre-stressed Orthotropic Layer and Pre-stressed Orthotropic Half-plane under the Action of a Moving Load. Int. J. Solids. Struct., 45 (2008), 4222-4235. 
[17] DAI, Z.-J., Z.-B. KuAng, S.-X. ZhaO. Rayleigh Waves in a Double Porosity Halfspace. J. Sound Vib., 298 (2006), 319-332.

[18] DAI, Z.-J., Z.-B. KuAng. Love Waves in Double Porosity Media. J. Sound Vib., 296 (2006), 1000-1012.

[19] KE, L.-L., Y.-S. WANG, Z.-M. ZHANG. Love Waves in an Inhomogeneous Fluid Saturated Porous Layered Half-space with Linearly Varying Properties. Soil Dyn. Earthq. Eng., 26 (2006), 574-581.

[20] Son, M. S., Y. J. KAng. Propagation of Shear Waves in a Poroelastic Layer constrained between Two Elastic Layers. Appl. Math. Model., 36 (2012), 3685-3695.

[21] Singh, A. K., A. LaKshman. Effect of Loosely Bonded Undulated Boundary Surfaces of Doubly Layered Half-space on the Propagation of Torsional Wave. Mech. Res. Commun., 73 (2016), 91-206.

[22] MurTy, S. A Theoretical Model for the Attenuation and Dispersion of Stoneley Waves at the Loosely-bonded Interface of Elastic Half-spaces. Phys. Earth Planet. Inter. 11 (1975), 65-79.

[23] MurTy, G. S. Reflection, Transmission and Attenuation of Elastic Waves at a Loosely Bonded Interface of Two Half-spaces. Geophys. J. R. Astron. Soc., 44 (1976), 389-404.

[24] Singh, A. K., Z. Parveen, A. Das, A. Chattopadhyay. Influence of Looselybonded Sandwiched Initially Stressed Visco-elastic Layer on Torsional Wave Propagation. J. of Mech., DOI 10.1017/jmech.2016.107.

[25] BIOT, M. A. Mechanics of Incremental Deformations, New York, John Wiley and Sons In., 1965.

[26] Prosser, W. H., R. E. Green. Characterization of the Nonlinear Elastic Properties of Graphite/epoxy Composites using Ultrasound. J. Reinf. Plast. Compos., 9 (1990), No. 2, 162-173.

[27] Batugin, S. A., R. K. Nirenburg. Approximate Relation between the Elastic Constants of Anisotropic Rocks and the Anisotropy Parameters. J. Min. Sci., 8 (1972), 5-9.

[28] Gubbins, D. Seismology and Plate Tectonics, Cambridge, Cambridge University Press, 1990. 